\title{
Comparison of tissue transglutaminase 2 and bone biological markers osteocalcin, osteopontin and sclerostin expression in human osteoporosis and osteoarthritis
}

\author{
Chiara Tarquini ${ }^{1,3} \cdot$ Rosanna Mattera $^{2} \cdot$ Francesca Mastrangeli $^{1,3} \cdot$ Sara Agostinelli $^{1}$. \\ Amedeo Ferlosio ${ }^{1} \cdot$ Roberto Bei $^{2} \cdot$ Augusto Orlandi $^{1,4} \cdot$ Umberto Tarantino $^{3,4}$
}

Received: 1 April 2016 / Accepted: 22 June 2016 / Published online: 29 June 2016

(c) Springer-Verlag Wien 2016

\begin{abstract}
Osteoporosis (OP) and osteoarthritis (OA) are the most common joint diseases, with a high incidence in the elderly population. OP is characterized by trabecular bone remodeling and reabsorption, whereas articular cartilage and subchondral bone remodeling are major features of OA. Although classically considered as independent or even conflicting processes, clinical coexistence of OP and OA was recently described. Transglutaminase 2 (TG2) expression is considered a biomarker of OA, but its role in osteoporotic bone remodeling is still uncertain. We investigated TG2 and bone biological markers (Osteocalcin, Osteopontin, and Sclerostin) in osteoporotic and osteoarthritic osteocartilagineous tissue $(n=54)$ and human chondrocyte cultures in vitro by immunohistochemistry, immunofluorescence and RT-PCR. Histomorphometric evaluation of bone trabecular remodeling was also performed. In cartilage, TG2 expression was faint in control and OP and significantly less than in $\mathrm{OA}$ and $\mathrm{OP}+\mathrm{OA}$ chondrocytes; the opposite was found for Osteocalcin, whereas Osteopontin
\end{abstract}

Handling Editors: S. Beninati, M. Piacentini, C.M. Bergamini.

C. Tarquini and R. Mattera contributed equally to the paper. A. Orlandi and U. Tarantino contributed equally to the paper.

Augusto Orlandi

orlandi@uniroma2.it

1 Anatomic Pathology, Department of Biomedicine and Prevention, Tor Vergata University of Rome, Rome, Italy

2 General Pathology, Department of Clinical Sciences and Translational Medicine, Tor Vergata University of Rome, Rome, Italy

3 Department of Orthopedics and Traumatology, Tor Vergata University of Rome, Rome, Italy

4 Policlinic of Tor Vergata of Rome, Rome, Italy and Sclerostin expression was similar. In the subchondral trabecular bone, osteocytes/osteoblasts TG2 expression was slight and similar comparing control, OP, OA, and $\mathrm{OP}+\mathrm{OA}$ group, whereas Osteocalcin and Osteopontin expression was lower in OP compared to control, OA and $\mathrm{OP}+\mathrm{OA}$. Increased TG2 and reduced Osteocalcin expression were maintained in human osteoarthritic chondrocytes in vitro. Histomorphometric analysis confirmed reduced trabecular bone mass in OP and OP + OA compared with OA patients. TG2 represented a suitable biomarker of osteoarthritic chondrocyte activation, whereas osteocalcin and osteopontin characterized osteoporotic osteocyte/osteoblast changes; differences were lost in OP + OA patients, suggesting careful consideration when coexistence of the two diseases occurs.

Keywords Osteoporosis · Osteoarthritis · Transglutaminase $2 \cdot$ Cartilage $\cdot$ Bone remodeling

\section{Introduction}

Osteoporosis (OP) and osteoarthritis (OA) are two main pathological processes affecting the elderly population. OP and OA are leading sources of physical disability and reduced quality of life, so resulting a relevant clinical problem and a major public health-economic challenge (Johnell and Kanis 2006; Dieppe and Lohmander 2005). OP is characterized by reduced mass and microarchitectural deterioration of bone tissue, with a consequent increase in bone fragility and susceptibility to fracture (Beil et al. 2008). OP is considered to be the result of the imbalance between bone formation and resorption (Beil et al. 2008). The main pathological feature of $\mathrm{OA}$ is the progressive degeneration and erosion of articular cartilage (Dieppe and 
Lohmander 2005). Subchondral bone sclerosis, remodeling of adjacent bone with osteophyte formation together with limited inflammation (synovitis) also occurs in OA (Dieppe and Lohmander 2005; Felson 2009). OA is considered a multifactorial disease and several risk factors contribute to its pathogenesis, such as aging, obesity, sex, joint malignment, genetic predisposition, and abnormal loading of the joints (Johnson and Hunter 2014). In OA, the chondrocyte dynamic equilibrium between the production of the extracellular matrix and its enzymatic degradation is lost and catabolic events prevail, leading to a progressive degradation of articular cartilage (Hinton et al. 2002). Concomitant changes in the bone and surrounding structures are also reported in OA patients (Hinton et al. 2002). Abnormal stress in OA may lead to subchondral microfractures and formation of reparative sclerotic bone, with a widely disorganized trabecular pattern (Power et al. 2010).

In the literature, several studies reported a controversial relationship between OP and OA. Many findings suggested an inverse association between OP and OA, with a protective effect of one disease on the other one (Cumming and Klineberg 1993; Dequeker and Johnell 1993; Vestergaard et al. 2009). Conversely, other relevant studies reported the possibility of coexistence of the two diseases, with increased risk of fracture and impaired bone quality in patients with OA (Tarantino et al. 2014; Bergink et al. 2003; Arden et al. 2006; Wright et al. 2011). Being pathogenetic pathways of OP and OA classically distinct, their causal or incidental coexistence in the same patient remains an unsolved question. It has been recently suggested that biochemical and cellular processes involving cartilage and bone intertwine and collectively damage joint compartment. In fact, clinical and experimental studies in OA patients showed changes in the subchondral bone (Berry et al. 2010; Goldring and Goldring 2010) and a cross-talk between cartilage and subchondral bone (Funck-Brentano and Cohen-Solal 2011). Therefore, loss of articular cartilage in OA may be linked to changes in the subjacent bone, through an altered load transmission and/or direct signaling (Karsdal et al. 2008). Transglutaminase 2 (TG2), also known as tissue transglutaminase, belongs to a family of enzymes that catalyze a calcium-dependent transamidation reaction. Transglutaminases generate covalent crosslinks of available substrate glutamine residues with primary amino groups and lead to changes in the structure and function of the implicated substrate proteins (Lorand and Graham 2003). TG2 is specifically implicated in a variety of cellular processes, such as differentiation, adhesion, migration, cell death, and survival (Fesus and Piacentini 2002; Lorand and Graham 2003). Several studies documented the upregulation of TG2 expression in chondrocytes and extracellular matrix of knee cartilage of OA patients and its contribution to pathologic cartilage matrix calcification (Johnson et al. 2001; Summey et al. 2002; Rosenthal et al. 1997). TG2 transamidation catalytic activity in joint cartilage was documented to be increased according to the severity of OA and in an age-dependent manner (Johnson et al. 2001; Rosenthal et al. 1997). Increased expression in damaged cartilage induced to consider TG2 as a biomarker to quantify pathological remodeling in OA patients (Orlandi et al. 2009; Tarantino et al. 2013). Pathological remodeling of subchondral trabecular bone is also documented in OP patients (Zhang et al. 2010), and biological markers of the severity of subchondral bone pathological reabsorption have been described. Osteocalcin $(\mathrm{OCN})$ is the major abundant non-collagenous bone protein, is synthesized and secreted by osteoblasts and is retained to regulate bone matrix formation and mineralization ( $\mathrm{Li}$ et al. 2016). OCN is also involved in pathological bone remodeling ( $\mathrm{Li}$ et al. 2016). Osteopontin (OPN) is a non-collagenous bone extracellular matrix protein secreted by various cells, including osteoblasts, osteoclasts, and chondrocytes (Denhardt and Noda 1998). OPN is retained to be involved in bone remodeling (Standal et al. 2004) but also contributes to the pathogenesis of OA (Pullig et al. 2000a; Gao et al. 2010; Matsui et al. 2009). Sclerostin (SOST) is an osteocyte-derived negative regulator of bone formation (Krause et al. 2010). SOST is also expressed by chondrocytes and reported to be implicated in the OP process of bone remodeling but also expressed in cartilage (Chan et al. 2011).

The aim of this work was to define the role of TG2 in the pathological articular remodeling in patients with OP and $\mathrm{OA}$ alone or in combination $(\mathrm{OP}+\mathrm{OA})$ and to correlate its expression with that of bone biological markers. The study of the expression of those markers in human cartilage and bone in a more personalized algorithm will be beneficial to address the knowledge of their specific role in OP and OA and will better predict the progression of diseases.

\section{Materials and methods}

\section{Patients population}

In this study, we selected 54 human femoral head samples, obtained from patients undergoing hip arthroplasty for primary OA or femoral neck fracture for OP at the Orthopaedic Department of Tor Vergata University of Rome. Clinical information is reported in Table 1. Before surgery, patients underwent to dual energy X-ray absorptiometry (DEXA) examination of lumbar spine and femoral neck to estimate the bone mineral density (BMD) (WHO 1994; Manenti et al. 2013). The percentage of BMD indicates the current value of the bone density in $\mathrm{g} / \mathrm{cm}^{2}$ and represents the best parameter to calculate the changes in bone density during follow-up of OP patients. According to the World Health Organization 
Table 1 Clinical features in OP, OA, and OP + OA patients

\begin{tabular}{llll}
\hline & OP & OA & OP + OA \\
\hline Number of patients & 20 & 19 & 15 \\
Mean age (years) & $76.95 \pm 2.62$ & $67.83 \pm 2.78$ & $72.07 \pm 2.57$ \\
Harris Hip Score & - & $48.5 \pm 4.31$ & $33.38 \pm 1.58$ \\
Bone mineral density & $-3.05 \pm 0.35$ & $1.79 \pm 0.26$ & $-2.8 \pm 0.24$ \\
\hline
\end{tabular}

Results are expressed as mean values \pm SEM

criteria (1994), the interpretation of the $T$ score is as follows: $T$ score >-1: normal, $T$ score between -1 and -2.5 : osteopenia, and $T$ score <-2.5: osteoporosis. Hip X-ray examination was performed to diagnose the radiological signs of OA (Ravaud et al. 1999). The Harris Hip Score (HHS) was also calculated in OA patients (Nilsdotter and Bremander 2011). Three different groups were assessed according to BMD, radiographic assessment, and histological features: OP $(n=20)$, OA $(n=19)$, and OP + OA patients $(n=15)$. For control group, anonymous sections of human femoral head samples ( $n=5$, mean age $52.6 \pm 2.85$ years) were obtained from the paraffin autopsy block archive of Anatomic Pathology of Tor Vergata University of patients with no history of joint disease and with macroscopically and microscopically normal osteocartilagineous structure.

\section{Histological and histomorphometric evaluation}

Formalin-fixed, decalcified, paraffin-embedded femoral head samples were cut in $5 \mu$ m-thick serial sections, stained with Haematoxylin \& Eosin and examined by light microscopy (Cassinelli et al. 2012). Articular cartilage and OA changes were evaluated according to international criteria (Collins and McElligotte 1960; Orlandi et al. 2009). Trabecular and subchondral bone was analyzed using Nikon Eclipse E600 optical microscope connected to a Nikon digital camera for the acquisition of the images. For each femoral head, at least 15 microscopic fields at $40 \times$ magnification of at least three different bone slides were evaluated. The images were analyzed using a BioQuant Osteo software (version 7.20.10; BIOQUANT Image Analysis Corporation, Nashville, USA) specific for the histomorphometric bone analysis. The following parameters were calculated: the bone volume (BV), the bone volume fraction as percentage of bone volume/total volume ratio (BV/TV), the bone surface (BS), the trabecular thickness (Tb. Th), the trabecular number $(\mathrm{Tb} . \mathrm{N})$, and the trabecular separation (Tb. Sp) (Harris 1969; Dempster et al. 2013).

\section{Immunohistochemical study}

For immunohistochemistry (Spagnoli et al. 1995), sections were incubated with rabbit polyclonal anti-TG2 (1:100 overnight; Covalab, Vinci-Biochem, Florence, Italy), antiOCN $(1 \mu \mathrm{g} / \mathrm{ml}$ for $1 \mathrm{~h}$; Abcam, Cambridge, UK), antiOPN ( $1 \mu \mathrm{g} / \mathrm{ml}$ for $1 \mathrm{~h}$; Sigma-Aldrich, Milan, Italy), and anti-SOST ( $1 \mu \mathrm{g} / \mathrm{ml}$ for $1 \mathrm{~h}$; Abcam, Cambridge, UK). The slides were then incubated with a goat anti-rabbit IgG peroxidase-conjugated secondary antibody (Sigma-Aldrich, Milan, Italy). After, the antigen-antibody complexes were visualized by 3-amino-9-ethylcarbazole (ScyTek, Logan, UT, USA) using Haematoxylin for counterstaining (Bei et al. 2004). Positive and negative controls were included to confirm the consistency of the analysis. Staining intensity in chondrocytes and osteocytes/osteoblasts was semiquantitatively graded using an arbitrary units system as follows: negative (0), weak and focal (0.5), weak diffuse (1), moderate (2), and high (3) (Orlandi et al. 2004). Each observation was repeated from two researchers. Inter-observer reproducibility $>95 \%$.

\section{Chondrocyte isolation and culture}

Human articular chondrocytes (HACs) were freshly isolated as reported (Jakob et al. 2003), with modifications. Briefly, small full thickness cartilage samples were finely minced within $1 \mathrm{~h}$ after surgery. For enzymatic digestion, cartilage pieces were pretreated with $0.25 \%$ trypsin $/ 1 \mathrm{mM}$ EDTA for $45 \mathrm{~min}$ at $37{ }^{\circ} \mathrm{C}$ and then incubated with $0.15 \%$ type II collagenase (Worthington Biochemical Corporation, Lakewood, NJ, USA) in Dulbecco's modified Eagle's medium (DMEM, Sigma-Aldrich) supplemented with $4.5 \mathrm{mg} / \mathrm{ml}$ glucose, $10 \mathrm{mM}$ HEPES buffer, $100 \mathrm{U} / \mathrm{ml}$ penicillin, $100 \mu \mathrm{g} / \mathrm{ml}$ streptomycin, $1 \mathrm{mM}$ sodium pyruvate, and $5 \%$ fetal bovine serum (FBS), for $22 \mathrm{~h}$ at $37^{\circ} \mathrm{C}$ with shaking. After filtration through a $100 \mu \mathrm{m}$ nylon mesh to remove undigested fragments, HACs were centrifugated and resuspended in DMEM supplemented with $2 \mathrm{mM}$ L-glutamine, $100 \mathrm{U} / \mathrm{ml}$ penicillin, $100 \mu \mathrm{g} / \mathrm{ml}$ streptomycin, $2.5 \mu \mathrm{g} / \mathrm{ml}$ amphotericin $\mathrm{B}$ and $10 \% \mathrm{FBS}$, and cultured in plastic dishes at $37{ }^{\circ} \mathrm{C}$ in a humidified atmosphere with $5 \% \mathrm{CO}_{2}$.

\section{Immunofluorescence studies}

For immunofluorescence assay (Cervelli et al. 2012), first passage HACs were fixed in methanol for $5 \mathrm{~min}$ at $-20^{\circ} \mathrm{C}$ and stained with rabbit polyclonal anti-TG2 (Covalab), anti-OCN (Abcam), anti-OPN (Sigma-Aldrich), and antiSOST (Abcam; 1:20 for $1 \mathrm{~h}$ ) and subsequently with rhodamine-labeled or fluorescein isothiocyanate-labeled antirabbit antibodies (Thermo Scientific Pierce, IL, USA), with negative controls. Nuclei were counterstained using Hoechst 33258 (Sigma-Aldrich). Cells were photographed with the Nikon ACT-1 camera controller software connected to Nikon Digital Camera DXM1200F, as reported (Cervelli et al. 2012). 


\section{Reverse-transcriptase-polymerase chain reaction (RT-PCR)}

After total RNA extraction, reverse-transcriptase-polymerase chain reaction (RT-PCR, Orlandi et al. 2005) was performed. Briefly, total RNA was extracted from cultured chondrocytes using Trizol reagent (Invitrogen, Life Technologies, Waltham, MA, USA), and RT-PCR was performed using Platinum Taq DNA Polymerase (Invitrogen) in an iCycler Thermal Cycler (Bio-Rad Laboratories, MI, Italy), according to the manufacturer's guidelines. Semiquantitative RT-PCR was performed using the following primers: for TG2, 5'-GAGGAGCTGGTCTTAGAGAGG-3' (sense) and 5'-CGGTCACGACACTGAAGGTG-3' (antisense) (NM_004613.3); for OCN, 5'-CAAAGGTGCAGCCTTTGTGTC-3' (sense) and 5'-TCACAGTCCGGATTGAGCTCA-3' (antisense) (NM_199173.4); for OPN, 5'-GCAGACCTGAC ATCCAGTACC-3' (sense) and 5'- GATGGCCTTGTATGC ACCATTC-3' (antisense) (NM_001251830.1); for SOST, 5'-ACCACCCCTTTGAGACCAAAG-3' (sense) and 5'-GGT CACGTAGCGGGTGAAGT-3' (antisense) (NM_025237.2); and for glyceraldehyde-3-phosphate dehydrogenase (GAPDH), 5'-ACGGATTTGGTCGTATTGG-3' (sense) and 5'-GATTTTGGAGGGATCTCGC-3' (antisense) (NM 002046). Results were normalized on housekeeping GAPDH gene as control. Experiments were performed in triplicate.

\section{Statistical analysis}

All results were expressed as the arithmetical mean \pm SEM. For the statistical evaluation, data were analyzed by the one-way analysis of variance (ANOVA) followed from a Bonferroni post hoc test and using the Student $t$ test. The differences were considered statistically significant for $p$ values $<0.05$.

\section{Results}

\section{Expression of TG2, OCN, OPN and SOST in human articular cartilage}

To assess the TG2 and other biomarkers expression in articular cartilage of control, OP, OA and OP + OA patients, immunohistochemical analysis was performed with specific antibodies. Representative images of TG2 and other biomarkers expression in cartilage of control, OP, OA, and OP + OA patients are shown in Fig. 1a. TG2 expression was faint and almost absent in control and OP and reduced compared with OA and OP + OA chondrocytes ( $p<0.01$; Fig. 1b). Semiquantitative analysis also documented the increased OCN expression in control and OP compared with OA and $\mathrm{OP}+\mathrm{OA}$ chondrocytes ( $p<0.01$ and $p<0.05$, respectively), without differences between $\mathrm{OA}$ and $\mathrm{OP}+\mathrm{OA}$ patients. No significant differences in OPN and SOST cartilage expression among different groups were observed (Fig. 1).

\section{Expression of TG2 and bone biological markers in trabecular bone}

We evaluated the expression of TG2 and other biomarkers in osteocytes/osteoblasts of subchondral trabecular bone (Fig. 2a). TG2 expression was slight and almost similar comparing all groups of patients in trabecular bone. OCN expression was lower in OP than in control, OA and OP + OA osteocytes/osteoblasts ( $p<0.01$; Fig. 2b). Increased OCN expression was also observed in $\mathrm{OP}+\mathrm{OA}$ compared to control and OA osteocytes/osteoblasts ( $p<0.01$; Fig. 2b). Furthermore, OPN expression was decreased in OP compared to control and OA $(p<0.05)$ and compared to OP + OA trabecular bone $(p<0.01$; Fig. 2b). No significant difference of SOST expression was found (Fig. 2b). Finally, SOST expression was much more marked in osteocytes/osteoblasts than in chondrocytes (Figs. 1a, 2a).

\section{Clinical and histomorphometric finding}

Histomorphometric analysis data from OP, OA and $\mathrm{OP}+\mathrm{OA}$ groups are reported in Table 2. Volumetrical analysis showed significant differences in BV value of femoral head samples, in particular, OA patients presented the highest BV compared to OP patients $(p<0.002)$. Statistically significant difference was also found comparing $\mathrm{BV}$ values of $\mathrm{OA}$ and $\mathrm{OP}+\mathrm{OA}$ patients $(p \ll 0.001)$. There were slight difference of $\mathrm{BV}$ and $\mathrm{BV} / \mathrm{TV}$ parameters between OP and OP + OA patients $(p<0.05)$, demonstrating that the amount of bone present in the subjects of the two groups can be considered almost similar.

Trabecular analysis also showed $\mathrm{Tb}$. Th value higher in OA patients than in the other groups, and it was reduced in those groups that presented the lowest BMD value (Table 1) as in $\mathrm{OP}$ and $\mathrm{OP}+\mathrm{OA}$ patients. The $\mathrm{Tb}$. $\mathrm{N}$ value was reduced in OP compared to OA patients $(p<0.002)$, with no significant between OP and OP + OA patients. Tb.Sp values showed an increased trabecular separation in OP compared to OA patients $(p \ll 0.001)$. OP and OP + OA patients did not show differences in $\mathrm{Tb}$. Th, Tb. N., and Tb. Sp values. All histomorphometric parameters were in line with BMD and X-ray differences among the groups (Table 1).

\section{Expression of TG2 and bone biological markers in human articular chondrocytes in vitro}

Freshly isolated HACs were plated onto culture dishes. As shown in Fig. 3a, HACs from OP and OA cartilage 

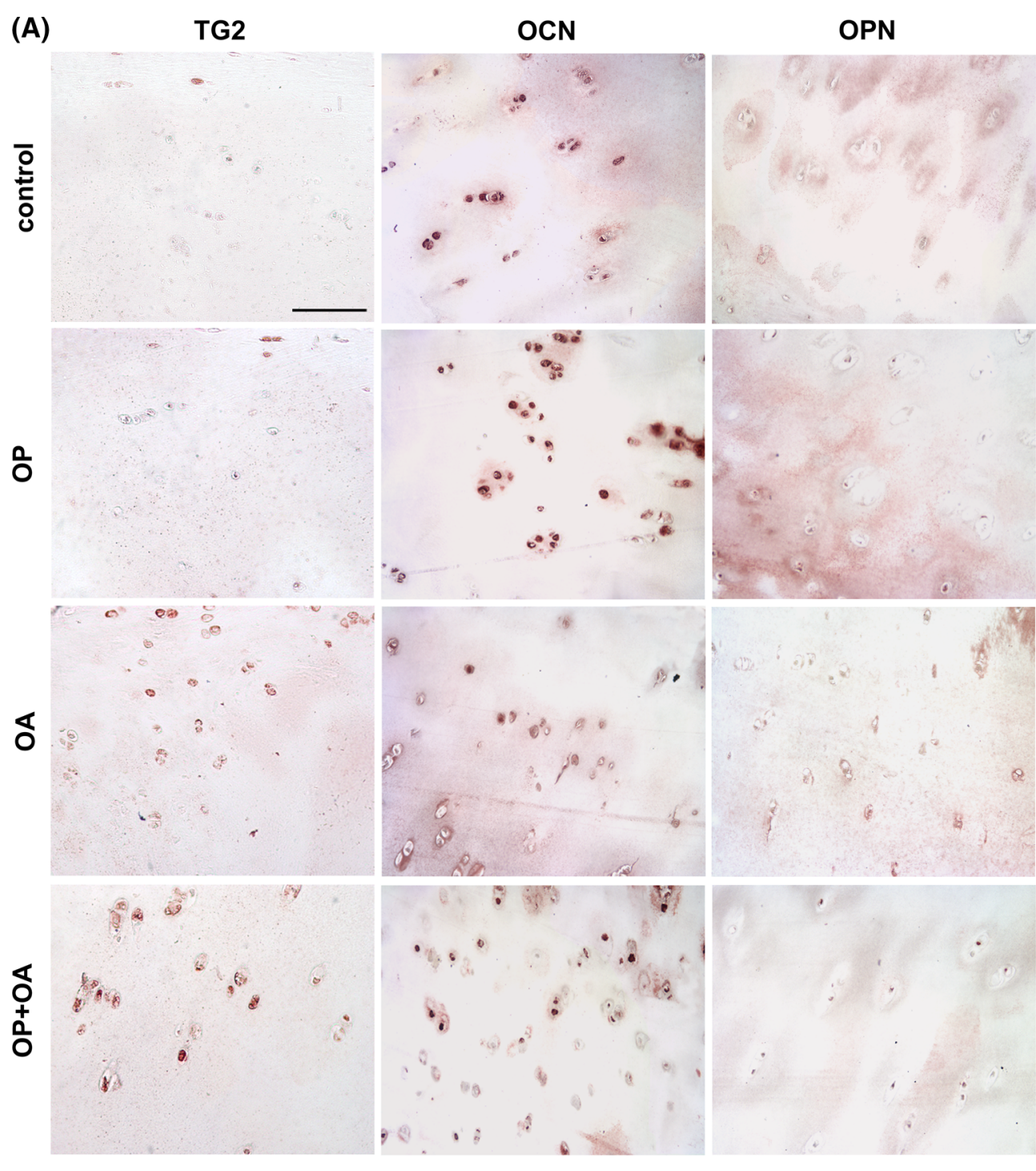

SOST

(B)
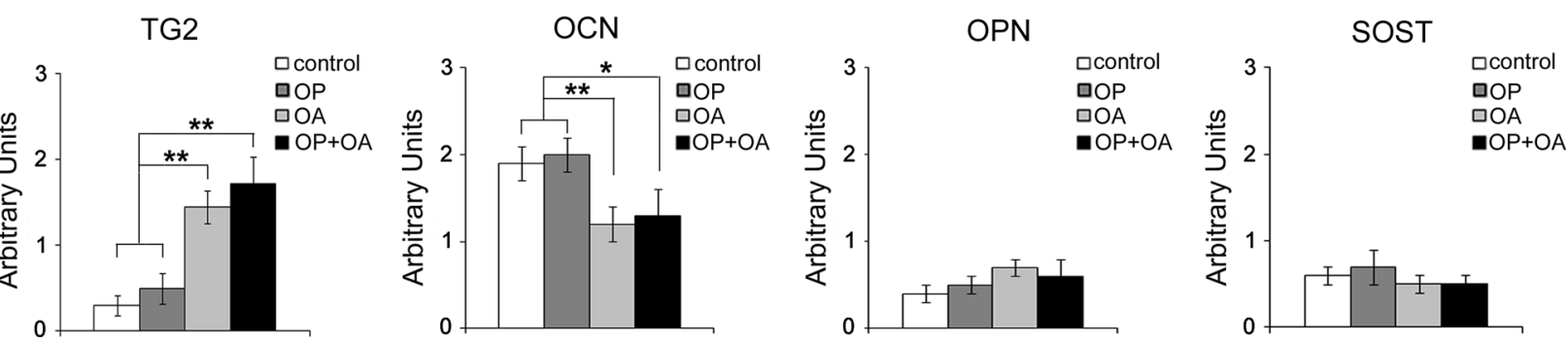

Fig. 1 TG2 and bone biological markers in human articular cartilage. a Representative images of TG2, OCN, OPN and SOST expressions in chondrocytes of human femoral cartilage of control, OP, OA and OP + OA patients. b Bar graphs show semiquantitative evaluation of

TG2, OCN, OPN, and SOST immunostainings; 3-amino-9-ethylcarbazole was used as chromogen. Results are expressed as mean values \pm SEM. ANOVA: $p<0.01$. ${ }^{*} p<0.05 ; * *<0.01$ at Student's $t$ test; Scale bar $100 \mu \mathrm{m}$

exhibited both a polygonal-to-elongate spindle cell morphology in vitro. Immunofluorescence staining (Fig. 3b) documented a reduced cytoplasmic TG2 expression in OP compared to OA chondrocytes. Conversely, OCN expression was higher in OP than that in OA HACs. HACs from

OP and OA cartilage showed a faint cytoplasmic OPN and SOST positivity, with no evident difference. Expression of TG2 and other biomarkers in HACs from OP + OA cartilage was almost similar to that of OA chondrocytes (data not shown). 

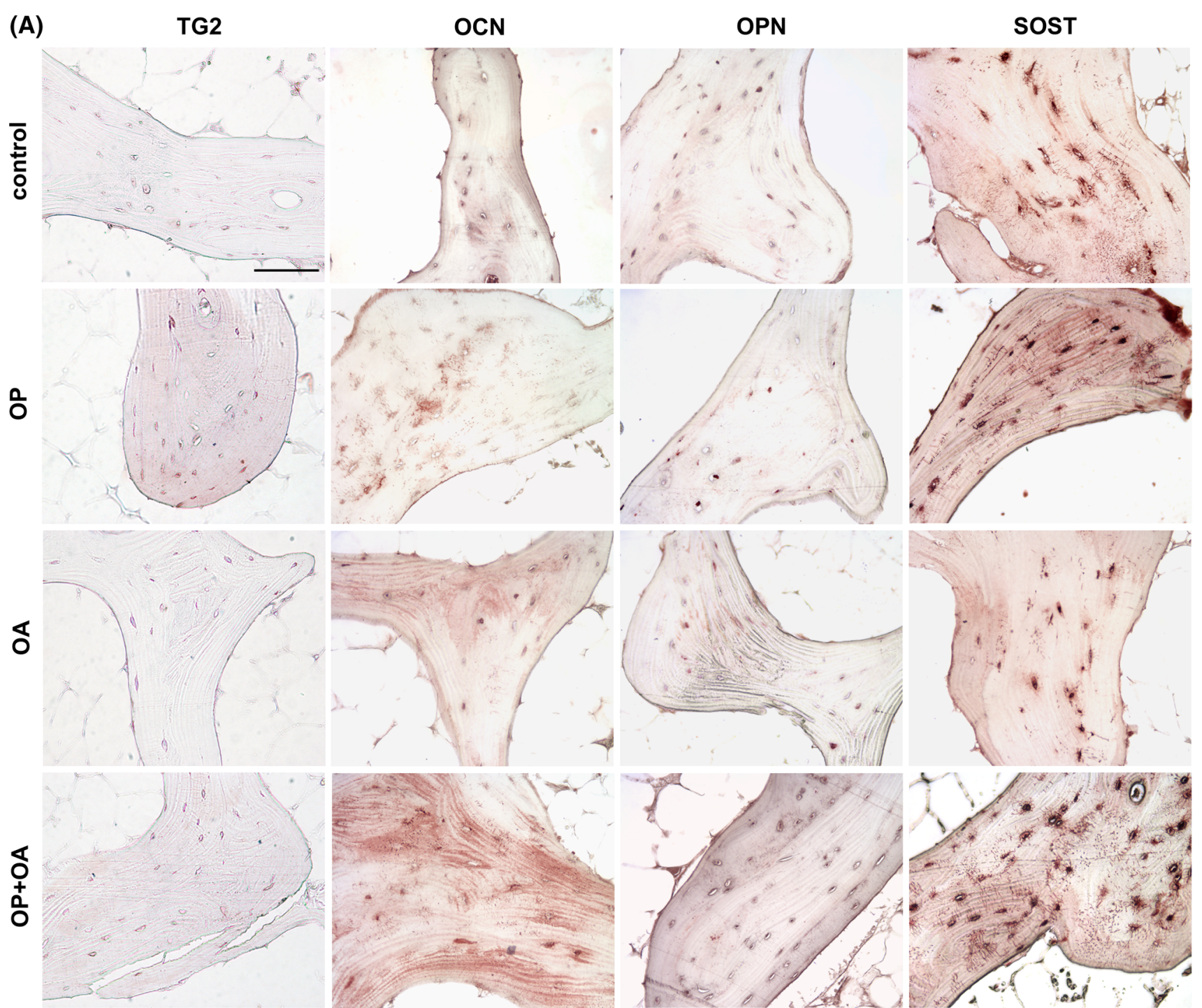

(B)
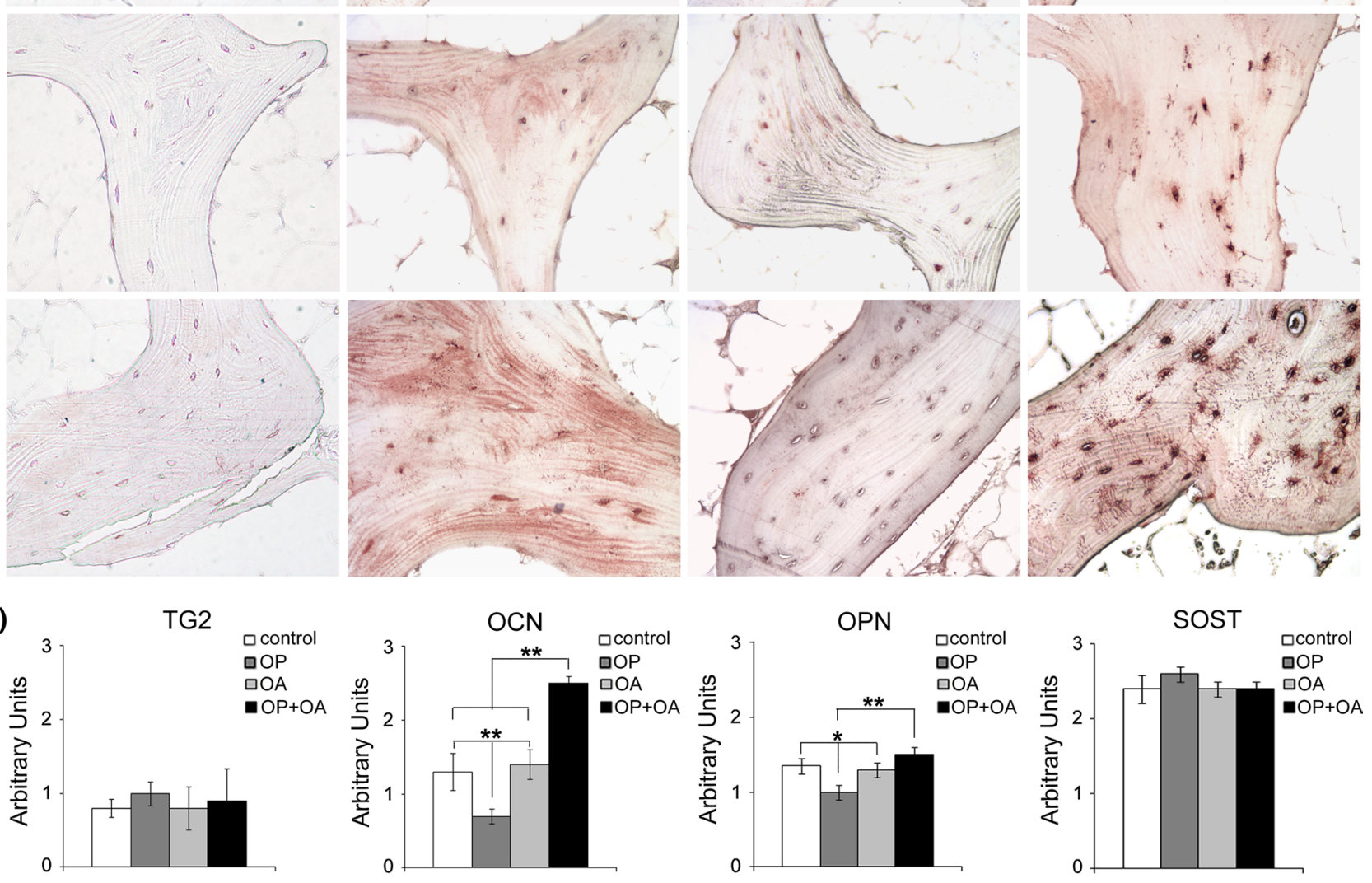

Fig. 2 TG2 and bone biological markers in human trabecular bone. a Representative images of TG2, OCN, OPN and SOST immunostaining in human femoral trabecular bone of control, OP, OA and $\mathrm{OP}+\mathrm{OA}$ patients. b Bar graphs show semiquantitative evaluation of

We investigated TG2, OCN, OPN, and SOST mRNA expression levels of OP and OA HACs by RT-PCR (Fig. 4a). Gene transcription analysis confirmed in vivo data, in particular, the increase of TG2 transcript levels in OA compared to OP and the reduction of OCN in OA compared to OP HACs (Fig. 4b).

TG2, OCN, OPN and SOST osteocytes/osteoblasts; 3-amino-9-ethylcarbazole is used as chromogen. Results are expressed as mean values \pm SEM. ANOVA: OCN, $p<0.0001$; OPN, $p<0.05$. ${ }^{*} p<0.05$; $* * p<0.01$ at Student's $t$ test; Scale bar $100 \mu \mathrm{m}$

\section{Discussion}

In this study, we provided new details about the expression of TG2 and bone biological markers in cartilage and trabecular bone of OP and OA patients. We documented that an increased chondrocyte TG2 expression characterized 
Table 2 Histomorphometric features evaluated in femoral head trabecular bone in OP, OA and OP + OA patients

\begin{tabular}{|c|c|c|c|c|c|c|}
\hline & & $\mathrm{OP}$ & & $\mathrm{OA}$ & & $\mathrm{OP}+\mathrm{OA}$ \\
\hline BV & & $3.4 \pm 0.1$ & & $6.2 \pm 0.3$ & & $3.8 \pm 0.2$ \\
\hline $\mathrm{BV} / \mathrm{TV}$ & & $20.5 \pm 0.8$ & & $37.2 \pm 1.5$ & & $23.2 \pm 1.5$ \\
\hline $\mathrm{BS}$ & & $80.7 \pm 3.1$ & & $92.2 \pm 4.2$ & & $78.3 \pm 4.4$ \\
\hline Tb. Th $(\mu \mathrm{m})$ & & $74 \pm 2.6$ & & $118.1 \pm 6.7$ & & $82.1 \pm 4.1$ \\
\hline Tb. $\mathrm{N}\left(\mathrm{mm}^{-1}\right)$ & & $2.7 \pm 0.06$ & & $3.5 \pm 0.2$ & & $2.9 \pm 0.2$ \\
\hline Tb. Sp $(\mu \mathrm{m})$ & & $310 \pm 9.2$ & & $189.2 \pm 9.7$ & & $307.6 \pm 29.8$ \\
\hline$p$ values & $\mathrm{BV}$ ( $p$ values $)$ & $\begin{array}{l}\mathrm{BV} / \mathrm{TV} \\
(p \text { values })\end{array}$ & $\mathrm{BS}$ ( $p$ values) & $\begin{array}{l}\text { Tb. Th }(\mu \mathrm{m})(p \\
\text { values })\end{array}$ & $\begin{array}{l}\text { Tb. } \mathrm{N}\left(\mathrm{mm}^{-1}\right) \\
(p \text { values })\end{array}$ & $\begin{array}{l}\text { Tb. Sp }(\mu \mathrm{m}) \\
(p \text { values })\end{array}$ \\
\hline OP vs OA & $<0.002$ & $<0.002$ & $<0.007$ & $\ll 0.001$ & $\ll 0.001$ & $\ll 0.001$ \\
\hline $\mathrm{OP}$ vs $\mathrm{OP}+\mathrm{OA}$ & $<0.05$ & $<0.05$ & $<0.65$ & $<0.88$ & $<0.44$ & $<0.93$ \\
\hline $\mathrm{OA}$ vs $\mathrm{OP}+\mathrm{OA}$ & $\ll 0.001$ & $<0.002$ & $<0.009$ & $\ll 0.001$ & $<0.034$ & $<0.001$ \\
\hline
\end{tabular}

Results are expressed as mean values \pm SEM

human OA cartilage degeneration, but not trabecular bone tissue remodeling independently from the cause. This strengthens the role of TG2 as a biomarker of OA (Tarantino et al. 2013). TG2 is a best-characterized, skeletal tissue-related TGs (Kaartinen et al. 2002) and is expressed in bone, cartilage, and teeth. TG2 has been suggested to be involved in matrix maturation and stabilization (Chen and Mehta 1999). In mineralized tissues, several collagen subtypes, fibronectin, osteopontin, and bone sialoprotein are all substrates for TG2; and are assembled in polymeric forms in the presence of $\mathrm{TG}$, so participating to matrix stabilization and mineralization and contributing to osteoblast and chondrocyte differentiation (Kaartinen et al. 2002; Beninati et al. 1994; Mukherjee et al. 1995). Conversely, osteocalcin seems to have an inhibitory effect on TG2 activity (Kaartinen et al. 1997). In OA, chondrocytes undergo hypertrophy with an accelerated turnover leading successively to calcification of pericellular matrix in the sclerotic subchondral bone (Ryan and McCarty 1997). TG2 is expressed from hypertrophic chondrocytes and considered a relevant factor promoting calcification and ossification (Thomázy and Davies 1999). TG2 expression was also found in osteoblast-like bone cells in vitro, confirming its involvement in extracellular matrix calcification and bone cell adhesion (Kaartinen et al. 2002; Heath et al. 2001; Wozniak et al. 2000). An increased TG2 gene transcription and a reduced matrix mineralization of subchondral human osteoblasts compared to non-sclerotic osteoblast were also described in vitro (Sanchez et al. 2008). Furthermore, TG2 gene knockout was recently documented to be associated with a reduced cartilage destruction and an increased osteophyte formation in an experimental model of mouse knee OA (Orlandi et al. 2009). Altogether, these data further support a main role of TG2 during osteoarthritic joint cartilage remodeling (Tarantino et al. 2013).
OCN, OPN, and SOST are several bone biological markers of interest that have been used in describing bone remodeling process, in particular in osteoporotic patients. We found an increased OCN expression in cartilage of OP patients and conversely reduced OCN level in trabecular bone of the same patients when compared to OA and $\mathrm{OP}+\mathrm{OA}$ groups. OCN is 49 amino acids, non-collagenous protein of bone extracellular matrix, synthesized and secreted by osteoblasts ( $\mathrm{Li}$ et al. 2016). OCN is also produced in lesser amount by odontoblasts and hypertrophic chondrocytes (Zoch et al. 2016). OCN is considered a marker of mature osteoblasts, and its activity is retained to regulate bone matrix formation and remodeling. OCN contains three $\gamma$-carboxyglutamic acid residues that confer a greater affinity for $\mathrm{Ca}^{2+}$ and hydroxyapatite, supporting a main role in the mineralization process. Modulation of OCN expression was reported in OA cartilage and trabecular bone. In particular, elevated OCN level was found in OA bone and cartilage compared to their normal counterpart (Pullig et al. 2000b; Kuliwaba et al. 2000). OCN is also used as a clinical marker for bone turnover, but controversial results have been reported concerning OCN serum levels (Wanby et al. 2016; Eastell and Hannon 2008). Increased OPN expression has been observed in joints, plasma, and synovial fluid of patients with OA (Pullig et al. 2000a; Gao et al. 2010). However, other studies reported decreased OPN levels during OA progression (Matsui et al. 2009). We observed increased OPN expression in osteoarthritic and osteoarthritic/osteoporotic trabecular bone, confirming that OPN can be considered a more general marker of bone remodeling. OPN is a highly phosphorylated and glycosylated protein, secreted by many tissues and cell types (Standal et al. 2004). OPN is involved in several physiologic and pathologic events, including wound healing, inflammation, angiogenesis, immune response, cell survival, cancer development, and metastasis 
(A)

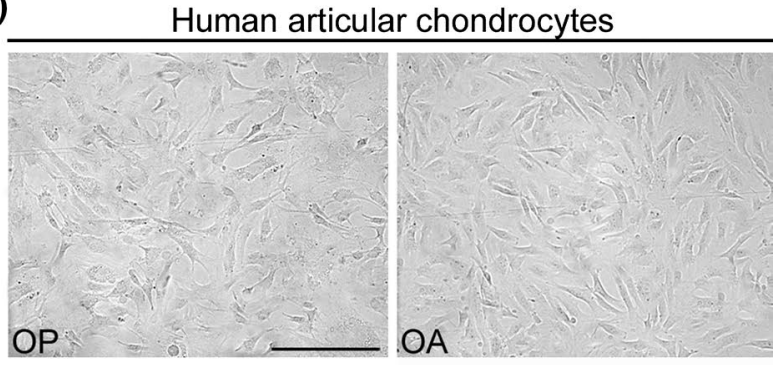

(B)
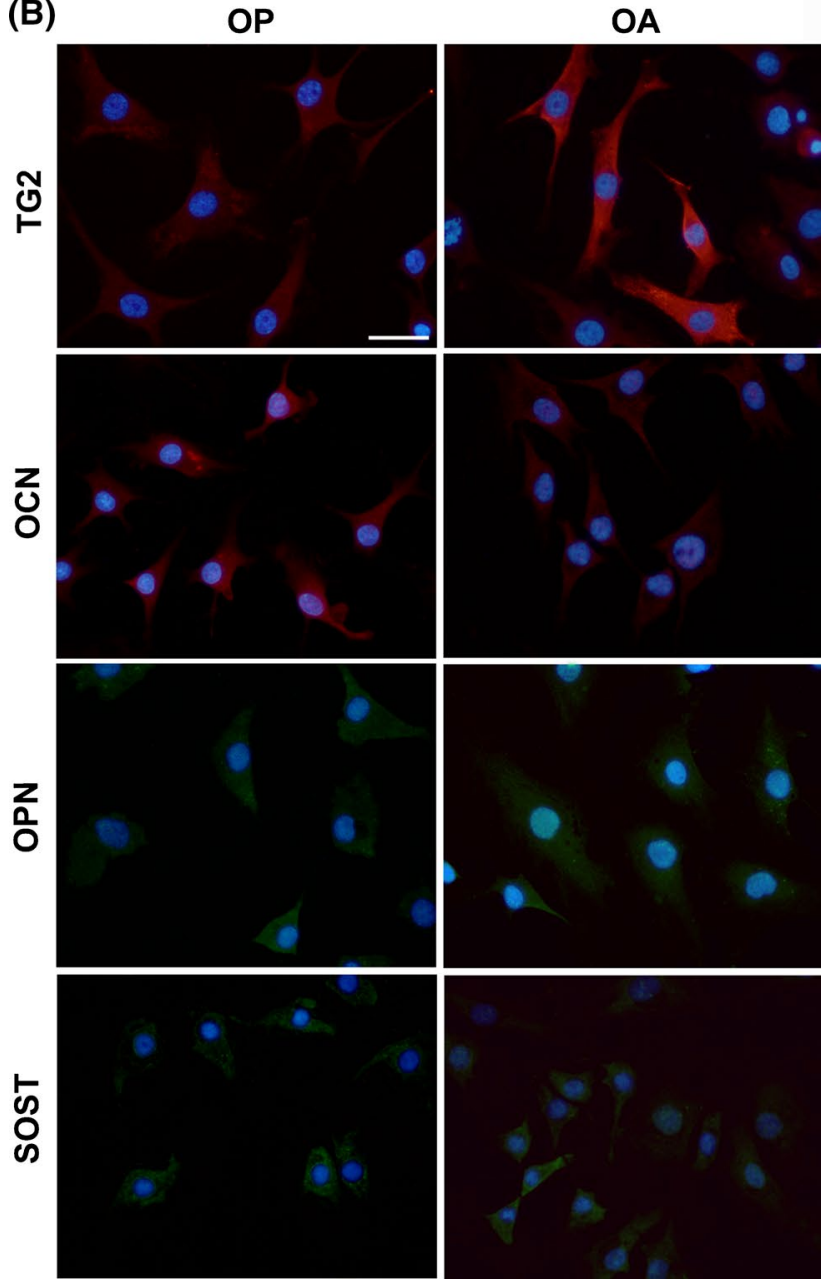

Fig. 3 Human chondrocyte morphology and TG2 and bone biological marker expression in vitro. a Phase contrast micrographs of human articular chondrocytes in serum cultures. Scale bar $150 \mu \mathrm{m}$; b TG2, OCN, OPN and SOST immunofluorescence staining cultured human articular chondrocytes of OP and OA patients. Nuclei are stained with Hoechst. Scale bar $50 \mu \mathrm{m}$

(Standal et al. 2004). OPN is also known as one of the major non-collagenous bone proteins, produced by osteoblast, osteoclast, chondrocytes, and synoviocytes (Denhardt and Noda 1998). OPN influences bone remodeling both by inhibiting mineral deposition and by promoting differentiation and activity of osteoclasts (Standal et al. 2004). OPN has been shown to bind calcium ions (Chen et al. 1992) and hydroxyapaytite crystal and found to be expressed in hypertrophic chondrocytes of the embryonic epiphyseal growth plates (McKee et al. 1992). Few studies focused on association between OPN levels and OP were performed. Some reports showed high serum OPN levels in osteoporotic patients (Chang et al. 2010; Wanby et al. 2016), suggesting that OPN may be considered a serum marker for the diagnosis of OP.

In the present study, we found no significant modulation of SOST expression in cartilage and bone of OP and OA alone or in combination. SOST is specifically expressed by osteocytes and belongs to the differential screeningselected gene aberrative in neuroblastoma (DAN) family of secreted glycoproteins (Krause et al. 2010). SOST is involved in bone homeostasis, in particular and acts as a direct extracellular antagonist of canonical Wnt signaling by binding to lipoprotein receptor-related protein-5/6 (LRP5/6) (Krause et al. 2010; Semënov et al. 2005). Loss of function of SOST causes high bone mineral density in humans (Piters et al. 2010; Bhadada et al. 2013), while SOST overexpression in transgenic mice leads to reduced bone formation and osteopenia (Winkler et al. 2003). SOST can regulate mechanical and inflammatory bone remodeling (Robling et al. 2008; Heiland et al. 2010). Recently, it has been reported that SOST is also expressed by chondrocytes and contributes to chondrocytes hypertrophic differentiation and implicated in OA process (Chan et al. 2011), likely by promoting subchondral bone sclerosis and inhibiting cartilage degradation. Other studies showed an increased SOST expression in OA chondrocyte clusters (Roudier et al. 2013). SOST has also been suggested to be involved in bone damage in postmenopausal women and hip fracture (Ardawi et al. 2011; Sarahrudi et al. 2012; Wanby et al. 2016). Of course, quantification and monitoring of OA and OP can be more easily performed by the evaluation of patients' experience of pain, X-rays, and serum markers of bone reabsorption. Although collected from articular tissues obtained by highly invasive methods, the present data still represent a starting point for a better understanding of the complex pathogenetic mechanisms of osteoporotic and osteoarthritic diseases. Further studies are needed to detect and confirm the role of these markers in more easily obtainable biological samples, including synovial aspirations.

In conclusion, our results documented that the TG2 expression was significantly less in control and OP than in $\mathrm{OA}$ and $\mathrm{OP}+\mathrm{OA}$ chondrocytes, whereas the opposite was true for OCN, and OPN and SOST expression did not differ. In the subchondral trabecular bone, TG2 expression was slight and similar comparing control, OA, OP, and $\mathrm{OP}+\mathrm{OA}$ group, whereas OCN and OPN was lower in OP compared to control, OA and OP + OA osteocytes/ 
Fig. 4 Transcript levels of TG2 and bone biological markers in human articular chondrocytes. a Representative gel and, b semiquantitative RT-PCR analysis of TG2, OCN, OPN, and SOST in cultured OP and OA human articular chondrocytes; GAPDH was used for normalization. Results are expressed as mean values \pm SEM of three experiments. Student's $t$ test: $* p<0.01 ; * * p<0.001$

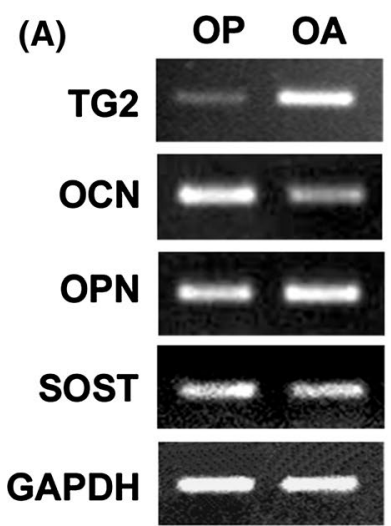

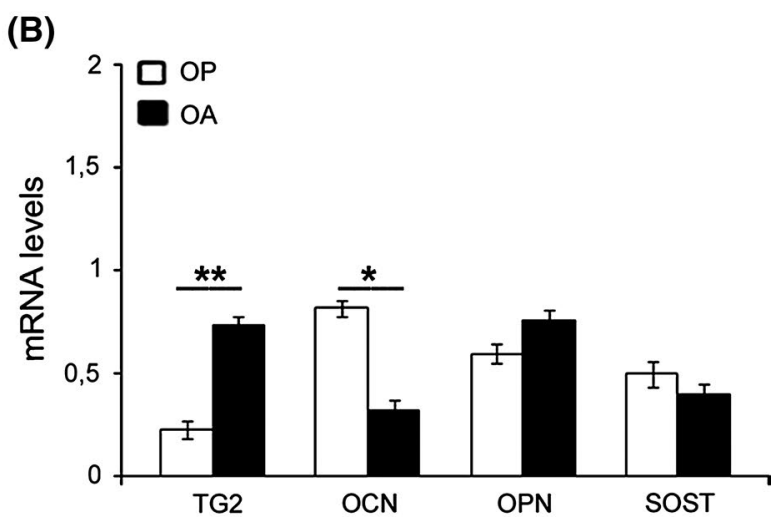

osteoblasts, and SOST expression marked in all groups. Those similar findings were observed in OP and OA HAC cultures, suggesting that this preclinical in vitro model is suitable to investigate intracellular pathogenetic pathways of OA degeneration. Although TG2 and OPN represent the most suitable biomarkers of OA chondrocyte activation and osteocyte/osteoblast changes, respectively, their meaning is lost in OP + OA patients, suggesting careful consideration in those patients with coexistence of the two diseases.

Acknowledgments The authors acknowledge ASI (Italian Space Agency) and University of Rome "Tor Vergata" (Spatial Biomedicine Center) for partially founding this study (number search DCDTE-2011-033). Thanks to S. Cappelli for technical assistance and to M. Feola and C. Rao for collection of clinical data.

\section{Compliance with ethical standards}

Conflict of interest The authors declare no conflict of interest.

Ethical approval All procedures performed in this study involving human participants were in accordance with the ethical standards of the University of "Tor Vergata", Ethic Committees and with the 1964 Helsinki Declaration and its later amendments or comparable ethical standards. Informed consent was obtained from all individual participants included in the study. This article does not contain any studies with animals performed by any of the authors.

\section{References}

Ardawi MS, Al-Kadi HA, Rouzi AA, Qari MH (2011) Determinants of serum sclerostin in healthy pre- and postmenopausal women. J Bone Miner Res 26:2812-2822. doi:10.1002/jbmr.479

Arden NK, Crozier S, Smith H, Anderson F, Edwards C, Raphael H, Cooper C (2006) Knee pain, knee osteoarthritis, and the risk of fracture. Arthritis Rheum 55:610-615. doi:10.1002/art.22088

Bei R, Budillon A, Masuelli L, Cereda V, Vitolo D, Di Gennaro E, Ripavecchia V, Palumbo C, Ionna F, Losito S, Modesti A, Kraus MH, Muraro R (2004) Frequent overexpression of multiple ErbB receptors by head and neck squamous cell carcinoma contrasts with rare antibody immunity in patients. J Pathol 204:317-325. doi:10.1002/path.1642

Beil FT, Seitz S, Priemel M, Barvencik F, von Domarus C, Rueger JM, Amling M, Pogoda P (2008) Pathophysiology and pathomorphology of osteoporosis. Eur J Trauma Emerg Surg 34:527-534. doi:10.1007/s00068-008-8201-y

Beninati S, Senger DR, Cordella-Miele E, Mukherjee AB, Chackalaparampil I, Shanmugam V, Singh K, Mukherjee BB (1994) Osteopontin: its transglutaminase-catalyzed posttranslational modifications and cross-linking to fibronectin. J Biochem 115:675-682

Bergink AP, van der Klift M, Hofman A, Verhaar JA, van Leeuwen JP, Uitterlinden AG, Pols HA (2003) Osteoarthritis of the knee is associated with vertebral and nonvertebral fractures in the elderly: the Rotterdam Study. Arthritis Rheum 49:648-657. doi:10.1002/art.11380

Berry PA, Maciewicz RA, Cicuttini FM, Jones MD, Hellawell CJ, Wluka AE (2010) Markers of bone formation and resorption identify subgroups of patients with clinical knee osteoarthritis who have reduced rates of cartilage loss. J Rheumatol 37:12521259. doi:10.3899/jrheum.091055

Bhadada SK, Rastogi A, Steenackers E, Boudin E, Arya A, Dhiman V, Bhansali A, Van Hul W (2013) Novel SOST gene mutation in a sclerosteosis patient and her parents. Bone 52:707-710. doi:10.1016/j.bone.2012.10.009

Cassinelli G, Zuco V, Petrangolini G, De Cesare M, Tortoreto M, Lanzi C, Cominetti D, Zaffaroni N, Orlandi A, Passeri D, Meco D, Di Francesco AM, Riccardi R, Bucci F, Pisano C, Zunino F (2012) The curative efficacy of namitecan (ST1968) in preclinical models of pediatric sarcoma is associated with antiangiogenic effects. Biochem Pharmacol 84:163-171. doi:10.1016/j. bcp.2012.04.005

Cervelli V, Scioli MG, Gentile P, Doldo E, Bonanno E, Spagnoli LG, Orlandi A (2012) Platelet-rich plasma greatly potentiates insulininduced adipogenic differentiation of human adipose-derived stem cells through a serine/threonine kinase Akt-dependent mechanism and promotes clinical fat graft maintenance. Stem Cells Transl Med 1:206-220. doi:10.5966/sctm.2011-0052

Chan BY, Fuller ES, Russell AK, Smith SM, Smith MM, Jackson MT, Cake MA, Read RA, Bateman JF, Sambrook PN, Little CB (2011) Increased chondrocyte sclerostin may protect against cartilage degradation in osteoarthritis. Osteoarthr Cartil 19:874885. doi:10.1016/j.joca.2011.04.014

Chang IC, Chiang TI, Yeh KT, Lee H, Cheng YW (2010) Increased serum osteopontin is a risk factor for osteoporosis in menopausal women. Osteoporos Int 21:1401-1409. doi:10.1007/ s00198-009-1107-7

Chen JS, Mehta K (1999) Tissue transglutaminase: an enzyme with a split personality. Int J Biochem Cell Biol 31:817-836. doi:10.1016/S1357-2725(99)00045-X

Chen Y, Bal BS, Gorski JP (1992) Calcium and collagen binding properties of osteopontin, bone sialoprotein, and bone acidic glycoprotein-75 from bone. J Biol Chem 267:24871-24878 
Collins DH, McElligotte TF (1960) Sulphate (35SO4) uptake by chondrocytes in relation to histological changes in osteoarthritic human articular cartilage. Ann Rheum Dis 19:318-330. doi:10.1136/ard.19.4.318

Cumming RG, Klineberg RJ (1993) Epidemiological study of the relation between arthritis of the hip and hip fractures. Ann Rheum Dis 52:707-710. doi:10.1136/ard.52.10.717

Dempster DW, Compston JE, Drezner MK, Glorieux FH, Kanis JA, Malluche H, Meunier PJ, Ott SM, Recker RR, Parfitt AM (2013) Standardized nomenclature, symbols, and units for bone histomorphometry: a 2012 update of the report of the ASBMR Histomorphometry Nomenclature Committee. J Bone Miner Res 28:2-17. doi:10.1002/jbmr.1805

Denhardt DT, Noda M (1998) Osteopontin expression and function: role in bone remodeling. $\mathrm{J}$ Cell Biochem Suppl 30-31:92-102. doi:10.1002/ (SICI) 1097-4644(1998)72:30/31+92:AID-JCB13>3.0.CO;2-A

Dequeker J, Johnell O (1993) Osteoarthritis protects against femoral neck fracture: the MEDOS study experience. Bone 14(Suppl 1):S51-S56. doi:10.1186/1471-2474-11-274

Dieppe PA, Lohmander LS (2005) Pathogenesis and management of pain in osteoarthritis. Lancet 365:965-973. doi:10.1016/ S0140-6736(05)71086-2

Eastell R, Hannon RA (2008) Biomarkers of bone health and osteoporosis risk. Proc Nutr Soc 67:157-162. doi:10.1017/ S002966510800699X

Felson DT (2009) Developments in the clinical understanding of osteoarthritis. Arthritis Res Ther 11:203. doi:10.1186/ar2531

Fesus L, Piacentini M (2002) Transglutaminase 2: an enigmatic enzyme with diverse functions. Trends Biochem Sci 27:534-539. doi:10.1016/S0968-0004(02)02182-5

Funck-Brentano T, Cohen-Solal M (2011) Crosstalk between cartilage and bone: when bone cytokines matter. Cytokine Growth Factor Rev 22:91-97. doi:10.1016/j.cytogfr.2011.04.003

Gao SG, Li KH, Zeng KB, Tu M, Xu M, Lei GH (2010) Elevated osteopontin level of synovial fluid and articular cartilage is associated with disease severity in knee osteoarthritis patients. Osteoarthr Cartil 18:82-87. doi:10.1016/j.joca.2009.07.009

Goldring MB, Goldring SR (2010) Articular cartilage and subchondral bone in the pathogenesis of osteoarthritis. Ann N Y Acad Sci 1192:230-237. doi:10.1111/j.1749-632.2009.05240.x

Harris WH (1969) Traumatic arthritis of the hip after dislocation and acetabular fractures: treatment by mold arthroplasty. An endresult study using a new method of result evaluation. J Bone Jt Surg Am 51:737-755

Heath DJ, Downes S, Verderio E, Griffin M (2001) Characterization of tissue transglutaminase in human osteoblast-like cells. J Bone Miner Res 16:1477-1485. doi:10.1359/jbmr.2001.16.8.1477

Heiland GR, Zwerina K, Baum W, Kireva T, Distler JH, Grisanti M, Asuncion F, Li X, Ominsky M, Richards W, Schett G, Zwerina J (2010) Neutralisation of Dkk-1 protects from systemic bone loss during inflammation and reduces sclerostin expression. Ann Rheum Dis 69:2152-2159. doi:10.1136/ard.2010.132852

Hinton R, Moody RL, Davis A, Thomas S (2002) Osteoarthritis: diagnosis and therapeutic considerations. Am Fam Physician 65:841-848

Jakob M, Démarteau O, Schäfer D, Stumm M, Heberer M, Martin I (2003) Enzymatic digestion of adult human articular cartilage yields a small fraction of the total available cells. Connect Tissue Res 44:173-180

Johnell O, Kanis JA (2006) An estimate of the worldwide prevalence and disability associated with osteoporotic fractures. Osteoporos Int 17:1726-1733. doi:10.1007/s00198-006-0172-4

Johnson VL, Hunter DJ (2014) The epidemiology of osteoarthritis. Best Pract Res Clin Rheumatol 28:5-15. doi:10.1016/j. berh.2014.01.004
Johnson K, Hashimoto S, Lotz M, Pritzker K, Terkeltaub R (2001) Interleukin-1 induces pro-mineralizing activity of cartilage tissue transglutaminase and factor XIIIa. Am J Pathol 159:149-163. doi:10.1016/S0002-9440(10)61682-3

Kaartinen MT, Pirhonen A, Linnala-Kankkunen A, Mäenpää PH (1997) Transglutaminase-catalyzed cross-linking of osteopontin is inhibited by osteocalcin. J Biol Chem 272:22736-22741. doi:10.1074/jbc.272.36.22736

Kaartinen MT, El-Maadawy S, Räsänen NH, McKee MD (2002) Tissue transglutaminase and its substrates in bone. J Bone Miner Res 17:2161-2173. doi:10.1359/jbmr.2002.17.12.2161

Karsdal MA, Leeming DJ, Dam EB, Henriksen K, Alexandersen P, Pastoureau P, Altman RD, Christiansen C (2008) Should subchondral bone turnover be targeted when treating osteoarthritis? Osteoarthr Cartil 16:638-646. doi:10.1016/j.joca.2008.01.014

Krause C, Korchynskyi O, de Rooij K, Weidauer SE, de Gorter DJ, van Bezooijen RL, Hatsell S, Economides AN, Mueller TD, Löwik CW, ten Dijke P (2010) Distinct modes of inhibition by sclerostin on bone morphogenetic protein and Wnt signaling pathways. J Biol Chem 285:41614-41626. doi:10.1074/jbc. M110.153890

Kuliwaba JS, Findlay DM, Atkins GJ, Forwood MR, Fazzalari NL (2000) Enhanced expression of osteocalcin mRNA in human osteoarthritic trabecular bone of the proximal femur is associated with decreased expression of interleukin- 6 and interleukin-11 mRNA. J Bone Miner Res 15:332-341. doi:10.1359/ jbmr.2000.15.2.332

Li J, Zhang H, Yang C, Li Y, Dai Z (2016) An overview of osteocalcin progress. J Bone Miner Metab. doi:10.1007/s00774-015-0734-7

Lorand L, Graham RM (2003) Transglutaminases: crosslinking enzymes with pleiotropic functions. Nat Rev Mol Cell Biol 4:140-156. doi:10.1038/nrm1014

Manenti G, Capuani S, Fanucci E, Assako EP, Masala S, Sorge R, Iundusi R, Tarantino U, Simonetti G (2013) Diffusion tensor imaging and magnetic resonance spectroscopy assessment of cancellous bone quality in femoral neck of healthy, osteopenic and osteoporotic subjects at 3T: preliminary experience. Bone 55:7-15. doi:10.1016/j.bone.2013.03.004

Matsui Y, Iwasaki N, Kon S, Takahashi D, Morimoto J, Matsui Y, Denhardt DT, Rittling S, Minami A, Uede T (2009) Accelerated development of aging-associated and instability-induced osteoarthritis in osteopontin-deficient mice. Arthritis Rheum 60:23622371. doi:10.1002/art.24705

McKee MD, Glimcher MJ, Nanci A (1992) High-resolution immunolocalization of osteopontin and osteocalcin in bone and cartilage during endochondral ossification in the chicken tibia. Anat Rec 234:479-492. doi:10.1002/ar.1092340404

Mukherjee BB, Nemir M, Beninati S, Cordella-Miele E, Singh K, Chackalaparampil I, Shanmugam V, DeVouge MW, Mukherjee AB (1995) Interaction of osteopontin with fibronectin and other extracellular matrix molecules. Ann N Y Acad Sci 760:201-212. doi:10.1111/j.1749-6632.1995.tb44631.x

Nilsdotter A, Bremander A (2011) Measures of hip function and symptoms: Harris Hip Score (HHS), Hip Disability and Osteoarthritis Outcome Score (HOOS), Oxford Hip Score (OHS), Lequesne Index of Severity for Osteoarthritis of the Hip (LISOH), and American Academy of Orthopedic Surgeons (AAOS) Hip and Knee Questionnaire. Arthritis Care Res (Hoboken) 63(Suppl 11):S200-S207. doi:10.1002/acr.20549

Orlandi A, Ferlosio A, Ciucci A, Sesti F, Lifschitz-Mercer B, Gabbiani G, Spagnoli LG, Czernobilsky B (2004) Cellular retinolbinding protein-1 expression in endometrial stromal cells: physiopathological and diagnostic implications. Histopathology 45:511-517. doi:10.1111/j.1365-2559.2004.01963.x

Orlandi A, Ciucci A, Ferlosio A, Pellegrino A, Chiariello L, Spagnoli LG (2005) Increased expression and activity of matrix 
metalloproteinases characterize embolic cardiac myxomas. Am J Pathol 166:1619-1628. doi:10.1016/S0002-9440(10)62472-8

Orlandi A, Oliva F, Taurisano G, Candi E, Di Lascio A, Melino G, Spagnoli LG, Tarantino U (2009) Transglutaminase-2 differently regulates cartilage destruction and osteophyte formation in a surgical model of osteoarthritis. Amino Acids 36:755-763. doi:10.1007/s00726-008-0129-3

Piters E, Culha C, Moester M, Van Bezooijen R, Adriaensen D, Mueller T, Weidauer S, Jennes K, de Freitas F, Löwik C, Timmermans JP, Van Hul W, Papapoulos S (2010) First missense mutation in the SOST gene causing sclerosteosis by loss of sclerostin function. Hum Mutat 31:E1526-E1543. doi:10.1002/humu.21274

Power J, Poole KE, van Bezooijen R, Doube M, Caballero-Alías AM, Lowik C, Papapoulos S, Reeve J, Loveridge N (2010) Sclerostin and the regulation of bone formation: effects in hip osteoarthritis and femoral neck fracture. J Bone Miner Res 25:1867-1876. doi:10.1002/jbmr.70

Pullig O, Weseloh G, Gauer S, Swoboda B (2000a) Osteopontin is expressed by adult human osteoarthritic chondrocytes: protein and mRNA analysis of normal and osteoarthritic cartilage. Matrix Biol 19:245-255. doi:10.1016/S0945-053X(00)00068-8

Pullig O, Weseloh G, Ronneberger D, Käkönen S, Swoboda B (2000b) Chondrocyte differentiation in human osteoarthritis: expression of osteocalcin in normal and osteoarthritic cartilage and bone. Calcif Tissue Int 67:230-240. doi:10.1007/s002230001108

Ravaud P, Ayral X, Dougados M (1999) Radiologic progression of hip and knee osteoarthritis. Osteoarthr Cartil 7:222-229. doi: $10.1007 / \mathrm{s} 10067-015-3146-0$

Robling AG, Niziolek PJ, Baldridge LA, Condon KW, Allen MR, Alam I, Mantila SM, Gluhak-Heinrich J, Bellido TM, Harris SE, Turner CH (2008) Mechanical stimulation of bone in vivo reduces osteocyte expression of Sost/sclerostin. J Biol Chem 283:5866-5875. doi:10.1074/jbc.M705092200

Rosenthal AK, Derfus BA, Henry LA (1997) Transglutaminase activity in aging articular chondrocytes and articular cartilage vesicles. Arthritis Rheum 40:966-970. doi:10.1002/art.1780400526

Roudier M, Li X, Niu QT, Pacheco E, Pretorius JK, Graham K, Yoon BR, Gong J, Warmington K, Ke HZ, Black RA, Hulme J, Babij $P$ (2013) Sclerostin is expressed in articular cartilage but loss or inhibition does not affect cartilage remodeling during aging or following mechanical injury. Arthritis Rheum 65:721-731. doi: $10.1002 /$ art. 37802

Ryan LM, McCarty DJ (1997) Calcium pyrophosphate crystal deposition disease, pseudogout, and articular condrocalcinosis. In: Koopman W (ed) Arthritis and allied conditions: a textbook of rheumatology, 13th edn. Williams and Wilkins, Baltimore, pp 2103-2126

Sanchez C, Deberg MA, Bellahcène A, Castronovo V, Msika P, Delcour JP, Crielaard JM, Henrotin YE (2008) Phenotypic characterization of osteoblasts from the sclerotic zones of osteoarthritic subchondral bone. Arthritis Rheum 58:442-455. doi:10.1002/ art.23159

Sarahrudi K, Thomas A, Albrecht C, Aharinejad S (2012) Strongly enhanced levels of sclerostin during human fracture healing. J Orthop Res 30:1549-1555. doi:10.1002/jor.22129

Semënov M, Tamai K, He X (2005) SOST is a ligand for LRP5/LRP6 and a Wnt signaling inhibitor. J Biol Chem 280:26770-26775. doi:10.1074/jbc.M504308200
Spagnoli LG, Orlandi A, Marino B, Mauriello A, De Angelis C, Ramacci MT (1995) Propionyl-L-carnitine prevents the progression of atherosclerotic lesions in aged hyperlipemic rabbits. Atherosclerosis 114:29-44. doi:10.1016/0021-9150(94)05460-Z

Standal T, Borset M, Sundan A (2004) Role of osteopontin in adhesion, migration, cell survival and bone remodeling. Exp Oncol 26:179-184

Summey BT Jr, Graff RD, Lai TS, Greenberg CS, Lee GM (2002) Tissue transglutaminase localization and activity regulation in the extracellular matrix of articular cartilage. J Orthop Res 20:76-82. doi:10.1016/S0736-0266(01)00064-X

Tarantino U, Ferlosio A, Arcuri G, Spagnoli LG, Orlandi A (2013) Transglutaminase 2 as a biomarker of osteoarthritis: an update. Amino Acids 44:199-207. doi:10.1007/s00726-011-1181-y

Tarantino U, Celi M, Rao C, Feola M, Cerocchi I, Gasbarra E, Ferlosio A, Orlandi A (2014) Hip osteoarthritis and osteoporosis: clinical and histomorphometric considerations. Int J Endocrinol 2014:372021. doi:10.1155/2014/372021

Thomázy VA, Davies PJ (1999) Expression of tissue transglutaminase in the developing chicken limb is associated both with apoptosis and endochondral ossification. Cell Death Differ 6:146-154

Vestergaard P, Rejnmark L, Mosekilde L (2009) Osteoarthritis and risk of fractures. Calcif Tissue Int 84:249-256. doi:10.1007/ s00223-009-9224-z

Wanby P, Nobin R, Von SP, Brudin L, Carlsson M (2016) Serum levels of the bone turnover markers dickkopf-1, sclerostin, osteoprotegerin, osteopontin, osteocalcin and 25-hydroxyvitamin $\mathrm{D}$ in Swedish geriatric patients aged 75 years or older with a fresh hip fracture and in healthy controls. J Endocrinol Invest. doi: 10.1007/s40618-015-0421-5

WHO (1994) Assessment of fracture risk and its application to screening for postmenopausal osteoporosis. Report of a WHO Study Group. World Health Organ Tech Rep Ser 843:1-129

Winkler DG, Sutherland MK, Geoghegan JC, Yu C, Hayes T, Skonier JE, Shpektor D, Jonas M, Kovacevich BR, Staehling-Hampton K, Appleby M, Brunkow ME, Latham JA (2003) Osteocyte control of bone formation via sclerostin, a novel BMP antagonist. EMBO J 22:6267-6276. doi:10.1093/emboj/cdg599

Wozniak M, Fausto A, Carron CP, Meyer DM, Hruska KA (2000) Mechanically strained cells of the osteoblast lineage organize their extracellular matrix through unique sites of alphavbeta3-integrin expression. J Bone Miner Res 15:1731-1745. doi:10.1359/jbmr.2000.15.9.1731

Wright NC, Lisse JR, Walitt BT, Eaton CB, Chen Z (2011) Arthritis increases the risk for fractures-results from the Women's Health Initiative. J Rheumatol 38:1680-1688. doi:10.3899/ jrheum. 101196

Zhang ZM, Li ZC, Jiang LS, Jiang SD, Dai LY (2010) Micro-CT and mechanical evaluation of subchondral trabecular bone structure between postmenopausal women with osteoarthritis and osteoporosis. Osteoporos Int 21:1383-1390. doi:10.1007/ s00198-009-1071-2

Zoch ML, Clemens TL, Riddle RC (2016) New insights into the biology of osteocalcin. Bone 82:42-49. doi:10.1016/j. bone.2015.05.046 